\title{
186.
}

\section{ON THE DETERMINATION OF THE VALUE OF A CERTAIN DETERMINANT.}

[From the Quarterly Mathematical Journal, vol. II. (1858), pp. 163-166.]

Considering the determinant

$$
\left|\begin{array}{cccccc}
\theta, & 1, & . & . & . & \cdots \\
n, & \theta, & 2 & & & \\
\cdot & n-1, & \theta, & 3 & & \\
& & n-2, & \theta, & 4 &
\end{array}\right|
$$

let the successive diagonal minors be $U_{0}, U_{1}, U_{2}, \ldots U_{x} \ldots$, it is easy to find

$$
\begin{aligned}
& U_{0}=1, \\
& U_{1}=\theta \\
& U_{2}=\left(\theta^{2}-1\right)-(n-1) \\
& U_{3}=\theta\left(\theta^{2}-4\right)-3(n-2) \theta \\
& U_{4}=\left(\theta^{2}-1\right)\left(\theta^{2}-9\right)-6(n-3)\left(\theta^{2}-1\right)+3(n-3)(n-1),
\end{aligned}
$$

which in fact suggests the law, viz.

$$
\begin{aligned}
U_{x}= & (\theta+x-1)(\theta+x-3)(\theta+x-5) \ldots(\theta-x+5)(\theta-x+3)(\theta-x+1) \\
& -\frac{x(x-1)}{2}(n-x+1)(\theta+x-3)(\theta+x-5) \ldots(\theta-x+5)(\theta-x+3) \\
& +\frac{x(x-1)(x-2)(x-3)}{2.4}(n-x+1)(n-x+3)(\theta+x-5) \ldots(\theta-x+5) \\
& -\& c . \\
& \vdots \\
& +(-)^{s} \frac{x(x-1) \ldots(x-2 s+1)}{2.4 \ldots 2 s}(n-x+1)(n-x+3) \ldots(n-x+2 s-1) \\
& \quad(\theta+x-2 s-1)(\theta+x-2 s-3) \ldots(\theta-x+2 s+1)
\end{aligned}
$$

to $s=\frac{1}{2} x$ or $\frac{1}{2}(x-1)$, as $x$ is even or odd. 
And of course if $x$ denote the number of lines or columns of the determinant, then $U_{x}$ is the value of the determinant. This theorem, or a particular case of it, is due to Prof. Sylvester: I have not been able to find an easier demonstration than the following one, which, it must be admitted, is somewhat complicated. I observe that $U_{x}$ satisfies the equation

$$
U_{x}-\theta U_{x-1}+(x-1)(n-x+2) U_{x-2}=0 .
$$

Hence writing $x-1$ and $x-2$ for $x$, we have the system

$$
\begin{array}{rlr}
U_{x}-\theta U_{x-1}+(x-1)(n-x+2) & U_{x-2} & =0 \\
U_{x-1}- & \theta U_{x-2}+(x-2)(n-x+3) U_{x-3} & =0 \\
U_{x-2}- & \theta U_{x-3}+(x-3)(n-x+4) U_{x-4} & =0,
\end{array}
$$

or, eliminating $U_{x-1}$ and $U_{x-3}$,

$$
\begin{aligned}
U_{x}+\left\{(x-1)(n-x+2)+(x-2)(n-x+3)-\theta^{2}\right\} U_{x-2} \\
+(x-2)(x-3)(n-x+3)(n-x+4) U_{x-4}=0 .
\end{aligned}
$$

Suppose, for shortness,

$$
(\theta+x-1)(\theta+x-3)(\theta+x-5) \ldots(\theta-x+5)(\theta-x+3)(\theta-x+1)=H_{x},
$$

and assume

$$
U_{x}=A_{x, 0} H_{x}-A_{x, 1} H_{x-2} \ldots+(-)^{s} A_{x, 8} H_{x-2 s} \ldots,
$$

where $A_{x, 8}$ is independent of $\theta$, then

$$
\begin{array}{lll}
U_{x} & \text { contains the term }(-)^{s} A_{x, 8} & H_{x-28}, \\
U_{x-2} & \text { contains the term }(-)^{s} A_{x-2,8} & H_{x-28-2},
\end{array}
$$

which is to be multiplied by

$$
(x-1)(n-x+2)+(x-2)(n-x+3)-\theta^{2} .
$$

This multiplier may be written under the form

$$
\begin{gathered}
(x-1)(n-x+2)+(x-2)(n-x+3)-(x-2 s-1)^{2}-\left\{\theta^{2}-(x-2 s-1)^{2}\right\} \\
=M_{x, s}-\left\{\theta^{2}-(x-2 s-1)^{2}\right\}
\end{gathered}
$$

if, for shortness,

$$
M_{x, 8}=(x-1)(n-x+2)+(x-2)(n-x+3)-(x-2 s-1)^{2} .
$$

Now

$$
M_{x, s}-\left\{\theta^{2}-(x-2 s-1)^{2}\right\}
$$

multiplied into

$$
(-)^{8} A_{x-2,8} H_{x-28-2}
$$

C. III. 
gives rise to the terms

$$
(-)^{8} M_{x, s} A_{x-2, s} H_{x-2 s-2}-(-)^{s} A_{x-2,8} H_{x-2 s},
$$

(since $\left\{\theta^{2}-(x-2 s-1)^{2}\right\} H_{x-28-2}=H_{x-28}$ ), or, what is the same thing,

$$
\begin{aligned}
& -(-)^{s} M_{x, 8-1} A_{x-2,8-1} H_{x-28}-(-)^{s} A_{x-2,8} H_{x-28} \\
= & -(-)^{s}\left\{M_{x, 8-1} A_{x-2,8-1}+A_{x-2,8}\right\} H_{x-28},
\end{aligned}
$$

and moreover

$$
U_{x-4} \text { contains the term }(-)^{8} A_{x-4,8} H_{x-2 s-4},
$$

or, what is the same thing, $(-)^{8} A_{x-4,8-2} H_{x-2 s}$.

Hence we must have

$$
A_{x, 8}-\left(A_{x-2,8}+M_{x, 8-1} A_{x-2,8-1}\right)+(x-2)(x-3)(n-x+3)(n-x+4) A_{x-4,8-2}=0 .
$$

where

$$
M_{x, s-1}=(x-1)(n-x+2)+(x-2)(n-x+3)-(x-2 s+1)^{2} .
$$

This may be satisfied by assuming

for then

$$
A_{x, 8}=B_{x, s}(n-x+1)(n-x+3) \ldots(n-x+2 s-1)
$$

$$
\begin{aligned}
A_{x-2,8} & =B_{x-2,8}(n-x+3) \ldots(n-x+2 s-1)(n-x+2 s+1), \\
A_{x-2,8-1} & =B_{x-2,8-1}(n-x+3) \ldots(n-x+2 s-1), \\
(n-x+3)(n-x+4) A_{x-4, x-2} & =B_{x-4,8-2}(n-x+4)(n-x+3) \ldots(n-x+2 s-1),
\end{aligned}
$$

and consequently

$$
\begin{aligned}
& B_{x, 8}(n-x+1) \\
- & B_{x-2,8}(n-x+2 s+1) \\
- & B_{x-2,8-1} M_{s, x-1} \\
+ & B_{x-4,8-2}(x-2)(x-3)(n-x+4)=0 ;
\end{aligned}
$$

and if this equation be satisfied independently of $n$, we must have

$$
\begin{aligned}
& B_{x, 8}-\quad B_{x-2,8}-(2 x-3) B_{x-2,8-1}+(x-2)(x-3) B_{x-4,8-2}=0 \\
& B_{x, 8}-(2 s+1) B_{x-2,8}-\left\{5 x-8-(x-2 s+1)^{2}\right\} B_{x-2,8-1}+4(x-2)(x-3) B_{x-4,8-2}=0
\end{aligned}
$$

and these are both satisfied by

$$
B_{x, s}=\frac{x \cdot x-1 \ldots x-2 s+1}{2^{s} \cdot 1 \cdot 2 \cdot 3 \ldots s}
$$

in fact, substituting this value and omitting the factor

$$
\frac{(x-2)(x-3) \ldots(x-2 s+1)}{2^{s} .1 .2 .3 \ldots s},
$$


the first equation becomes

$$
x(x-1)-\quad(x-2 s)(x-2 s-1)-\quad(2 x-3) 2 s+4 s(s-1)=0,
$$

and the second equation becomes

$$
x(x-1)-(2 s+1)(x-2 s)(x-2 s-1)-\left\{5 x-8-(x-2 s+1)^{2}\right\} 2 s+16 s(s-1)=0,
$$

which are each of them an identical equation, the first being

and the second being

$$
\begin{aligned}
x^{2}-\quad x \\
-x^{2}+(4 s+1) x-2 s(2 s+1) \\
-4 s x+6 s \\
+4 s(s-1)=0
\end{aligned}
$$

$$
\begin{aligned}
& x^{2}-\quad x \\
&-(2 s+1)\left\{x^{2}-(4 s+1) x+2 s(2 s+1)\right\} \\
&+2 s\left\{x^{2}-(4 s+3) x+(2 s-1)^{2}+8\right\} \\
&+16 s(s-1)=0
\end{aligned}
$$

as may be easily verified.

Hence writing for $B_{x, s}$ its value and recapitulating, the equation

$$
\begin{aligned}
U_{x}+\{(x-1)(n-x+2)+(x-2) & \left.(n-x+3)-\theta^{2}\right\} U_{x-2} \\
+ & (x-2)(x-3)(n-x+3)(n-x+4) U_{x-4}=0
\end{aligned}
$$

is satisfied by

$$
U_{x}=A_{x, 0} H_{x}-A_{x, 1} H_{x-2} \ldots+(-)^{8} A_{x, 8} H_{x-28} \ldots \text { to } s=\frac{1}{2} x \text { or } \frac{1}{2}(x-1) \text {, as } x \text { is even or odd, }
$$

where

$$
\begin{aligned}
& H_{x}=(\theta+x-1)(\theta+x-3)(\theta+x-5) \ldots(\theta-x+5)(\theta-x+3)(\theta-x+1), \\
& A_{x, 8}=\frac{x(x-1) \ldots(x-2 s+1)}{2^{s} .1 .2 .3 \ldots s}(n-x+1)(n-x+3) \ldots(n-x+2 s-1),
\end{aligned}
$$

and since for $x=0,1,2,3$ the values of the expression $U_{x}$ coincide with those of the first four diagonal minors, the expression gives in general the value of the diagonal minor, or when $x$ denotes the number of lines or columns of the determinant, then the value of the determinant.

2, Stone Buildings, 1st April, 1857. 